\title{
ON INVESTIGATION OF SOME NON-LINEAR INTEGRAL BOUNDARY VALUE PROBLEM
}

\author{
I. VARGA
}

Received 07 November, 2018

\begin{abstract}
We suggest a constructive approach for the solvability analysis and approximate solution of certain types of partially solved Lipschitzian differential systems with mixed two-point and integral non-linear boundary conditions. The practical application of the suggested technique is shown on a numerical example.
\end{abstract}

2010 Mathematics Subject Classification: 34B15

Keywords: implicit differential systems, non-linear integral boundary conditions, parametrization technique, successive approximations

\section{Problem Setting}

This article uses the approach proposed in [2], [5], [4] in the case of the following non-linear boundary value problem with mixed two-point and integral restrictions

$$
\begin{gathered}
\frac{d x(t)}{d t}=f\left(t, x(t), \frac{d x(t)}{d t}\right), t \in[a, b], \\
g\left(x(a), x(b), \int_{a}^{b} h(s, x(s)) d s\right)=d .
\end{gathered}
$$

We suppose that $f:[a, b] \times D \times D_{1} \rightarrow \mathbb{R}^{n}$ is a continuous function defined on a bounded sets $D \subset \mathbb{R}^{n}, D^{1} \subset \mathbb{R}^{n}$ (domain $D:=D_{\rho}$ will be concretized later, see (1.8), $D^{1}$ is given ) and $d \in \mathbb{R}^{n}$ is a given vector. Moreover $f, g: D \times D \times D_{2} \rightarrow$ $\mathbb{R}^{n}$ and $h:[a, b] \times D \rightarrow \mathbb{R}^{n}$ are Lipschitzian in the following form

$$
\begin{gathered}
|f(t, u, v)-f(t, \widetilde{u}, \widetilde{v})| \leq K_{1}|u-\widetilde{u}|+K_{2}|v-\widetilde{v}|, \\
|g(u, w, p)-g(\widetilde{u}, \widetilde{w}, \widetilde{p})| \leq K_{3}|u-\widetilde{u}|+K_{4}|w-\widetilde{w}|+K_{5}|p-\widetilde{p}| \\
|h(t, u)-h(t, \widetilde{u})| \leq K_{6}|u-\widetilde{u}|
\end{gathered}
$$


for any $t \in[a, b]$ fixed, all $\{u, \widetilde{u}\} \subset D,\{v, \widetilde{v}\} \subset D^{1},\{w, \widetilde{w}\} \subset D,\{p, \widetilde{p}\} \subset D_{2}$, where

$$
D_{2}:=\left\{\int_{a}^{b} h(t, x(t)) d t: t \in[a, b], x \in D\right\}
$$

and $K_{1}-K_{5}$ are non-negative square matrices of dimension $n$. The inequalities between vectors are understood componentwise. A similar convention is adopted for the "absolute value", "max", "min" operations. The symbol $I_{n}$ stands for the unit matrix of dimension $n, r(K)$ denotes a spectral radius of a square matrix $K$.

By the solution of the problem (1.1), (1.2) we understand a continuously differentiable function with property (1.2) satisfying (1.1) on $[a, b]$.

We fix certain bounded sets $D_{a} \subset \mathbb{R}^{n}$ and $D_{b} \subset \mathbb{R}^{n}$ and focus on the solutions $x$ of the given problem with property $x(a) \in D_{a}$ and $x(b) \in D_{b}$. Instead of the nonlocal boundary value problem (1.1), (1.2), we consider the parameterized family of two-point "model -type " problems with simple separated conditions

$$
\begin{aligned}
\frac{d x(t)}{d t}= & f\left(t, x(t), \frac{d x(t)}{d t}\right), t \in[a, b], \\
& x(a)=z, x(b)=\eta,
\end{aligned}
$$

where $z=\left(z_{1}, z_{2}, \ldots, z_{n}\right), \eta=\left(\eta_{1}, \eta_{2}, \ldots, \eta_{n}\right)$ are considered as parameters.

If $z \in \mathbb{R}^{n}$ and $\rho$ is a vector with non-negative components, $O(z, \rho):=\left\{\xi \in \mathbb{R}^{n}:|\xi-z| \leq \rho\right\}$ stands for the componentwise $\rho$-neighbourhood of $z$. For given two bounded connected sets $D_{a} \subset \mathbb{R}^{n}$ and $D_{b} \subset \mathbb{R}^{n}$, introduce the set

$$
D_{a, b}:=(1-\theta) z+\theta \eta, z \in D_{a}, \eta \in D_{b}, \theta \in[0,1]
$$

and its componentwise $\rho$-neighbourhood by putting

$$
D=D_{\rho}:=O\left(D_{a, b}, \rho\right)=\underset{\xi \in D_{a, b}}{\cup} O(\xi, \rho)
$$

We suppose that

$$
r\left(K_{2}\right)<1, r(Q)<1
$$

where

$$
\begin{gathered}
Q:=\frac{3(b-a)}{10} K, \\
K=K_{1}+K_{2}\left[I_{n}-K_{2}\right]^{-1} K_{1}=\left[I_{n}-K_{2}\right]^{-1} K_{1} .
\end{gathered}
$$

On the base of function $f:[a, b] \times D \times D^{1} \rightarrow \mathbb{R}^{n}$ we introduce the vector

$$
\delta_{[a, b], D, D^{1}}(f):=\frac{1}{2}\left[\max _{(t, x, y) \in[a, b] \times D \times D^{1}} f(t, x, y)-\min _{(t, x, y) \in[a, b] \times D \times D^{1}} f(t, x, y)\right]
$$


and suppose that the $\rho$-neighbourhood in (1.8) is such that

$$
\rho \geq \frac{b-a}{2} \delta_{[a, b], D, D^{1}}(f) .
$$

\section{MAIN STATEMENTS}

The investigation of the solutions of parameterized problem (1.6) and (1.7) is connected with the properties of the following special sequence of functions well posed on the interval $t \in[a, b]$

$$
\begin{gathered}
x_{0}(t, z, \eta)=z+\frac{t-a}{b-a}[\eta-z]=\left[1-\frac{t-a}{b-a}\right] z+\frac{t-a}{b-a} \eta, t \in[a, b], \\
x_{m+1}(t, z, \eta)=z+\int_{a}^{t} f\left(s, x_{m}(s, z, \eta), \frac{d x_{m}(s, z, \eta)}{d s}\right) d s- \\
-\frac{t-a}{b-a} \int_{a}^{b} f\left(s, x_{m}(s, z, \eta), \frac{d x_{m}(s, z, \eta)}{d s}\right) d s+\frac{t-a}{b-a}[\eta-z], t \in[a, b], \\
m=0,1,2, \ldots,
\end{gathered}
$$

Theorem 1. Let assumptions (1.3)-(1.5) and (1.9) hold. Then, for all fixed $(z, \eta) \in$ $D_{a} \times D_{b}$ :

1. The functions of the sequence (2.2) are continuously differentiable functions on the interval $t \in[a, b]$, have values in the domain $D=D_{\rho}$ and satisfy the two-point separated boundary conditions (1.7).

2. The sequence of functions (2.2) in $t \in[a, b]$ converges uniformly as $m \rightarrow \infty$ to the limit function

$$
x_{\infty}(t, z, \eta)=\lim _{m \rightarrow \infty} x_{m}(t, z, \eta)
$$

satisfying the two-point separated boundary conditions (1.7).

3. The limit function $x_{\infty}(t, z, \eta)$ is the unique continuously differentiable solution of the integral equation

$$
\begin{gathered}
x(t)=z+\int_{a}^{t} f\left(s, x(s), \frac{d x(s)}{d s}\right) d s-\frac{t-a}{b-a} \int_{a}^{b} f\left(s, x(s), \frac{d x(s)}{d s}\right) d s+ \\
+\frac{t-a}{b-a}[\eta-z],
\end{gathered}
$$

i.e. it is the solution of the Cauchy problem for the modified system of integrodifferential equations :

$$
\frac{d x}{d t}=f\left(t, x, \frac{d x(t)}{d t}\right)+\frac{1}{b-a} \Delta(z, \eta), x(a)=z
$$


where $\Delta(z, \eta): D_{a} \times D_{b} \rightarrow \mathbb{R}^{n}$ is a mapping given by formula

$$
\Delta(z, \eta):=[\eta-z]-\int_{a}^{b} f\left(s, x_{\infty}(s, z, \eta), \frac{d x_{\infty}(s, z, \eta)}{d s}\right) d s .
$$

4.The following error estimate holds:

$$
\begin{gathered}
\left|x_{\infty}(t, z, \eta)-x_{m}(t, z, \eta)\right| \leqslant \\
\leqslant \frac{10}{9} \alpha_{1}(t, a, b-a) Q^{m}\left(1_{n}-Q\right)^{-1} \delta_{[a, b], D, D^{1}}(f),
\end{gathered}
$$

for any $t \in[a, b]$ and $m \geq 0$, where $\delta_{[a, b], D, D^{1}}(f)$ is given in (1.11) and

$$
\alpha_{1}(t, a, b-a)=2(t-a)\left(1-\frac{t-a}{b-a}\right), \alpha_{1}(t, a, b-a) \leq \frac{b-a}{2} .
$$

Proof. The validity of this statement can be established similarly to Theorem 1 in [4].

Theorem 2. Under the assumption of Theorem 1, the limit function $x_{\infty}(t, z, \eta)$ : $[a, b] \times D_{a} \times D_{b} \rightarrow \mathbb{R}^{n}$ defined by (2.3) is a continuously differentiable solution of the original BVP (1.1), (1.2) if and only if the pair of vectors $(z, \eta)$ satisfies the system of $2 n$ determining algebraic equations

$$
\left\{\begin{array}{l}
\Delta(z, \eta)=\eta-z-\int_{a}^{b} f\left(s, x_{\infty}(s, z, \eta), \frac{d x_{\infty}(s, z, \eta)}{d s}\right) d s=0, \\
\Lambda(z, \eta)=g\left(x_{\infty}(a, z, \eta), x_{\infty}(b, z, \eta), \int_{a}^{b} h\left(s, x_{\infty}(s, z, \eta)\right) d s\right)-d=0 .
\end{array}\right.
$$

Note, that similarly as in [3], the solvability of the determining system (2.9) on the base of (1.3)-(1.5) and (1.9) can be established by studying its $m$-th approximate versions:

$$
\left\{\begin{array}{l}
\Delta_{m}(z, \eta)=\eta-z-\int_{a}^{b} f\left(s, x_{m}(s, z, \eta), \frac{d x_{m}(s, z, \eta)}{d s}\right) d s=0, \\
\Lambda_{m}(z, \eta)=g\left(x_{m}(a, z, \eta), x_{m}(b, z, \eta), \int_{a}^{b} h\left(s, x_{m}(s, z, \eta)\right) d s\right)-d=0 .
\end{array}\right.
$$

where $m$ is fixed.

Lemma 1. Under the assumptions of Theorem 1, for the exact and approximate determining functions defined by (2.9) and (2.10) for any $(z, \eta) \in D_{a} \times D_{b}$ and $m \geq 1$ 
hold the following estimates:

$$
\begin{aligned}
\left|\Delta(z, \eta)-\Delta_{m}(z, \eta)\right| & \frac{10(b-a)^{2}}{27} K Q^{m}\left(1_{n}-Q\right)^{-1} \delta_{[a, b], D, D_{1}}(f), \\
\left|\Lambda(z, \eta)-\Lambda_{m}(z, \eta)\right| \leq & \frac{5(b-a)}{9}\left[K_{3}+K_{4}+\right. \\
& \left.+(b-a) K_{5} K_{6}\right] Q^{m}\left(1_{n}-Q\right)^{-1} \delta_{[a, b], D, D_{1}}(f),
\end{aligned}
$$

where the matrix $Q$ and the vector $\delta_{[a, b], D, D_{1}}(f)$ are given respectively in (1.10) and (1.11).

Proof. Let us fix an arbitrary $(z, \eta) \in D_{a} \times D_{b}$. Direct computation gives that

$$
\int_{a}^{b} \alpha_{1}(t, a, b-a) d t=\frac{(b-a)^{2}}{3} .
$$

On the base of (1.1) and (1.3), when $u \neq \widetilde{u}$, we have

$$
|f(t, u, v)-f(t, \widetilde{u}, \widetilde{v})| \leq K|u-\widetilde{u}|,
$$

where matrix $K$ is given in (1.10). Taking into account (2.7) we obtain

$$
\begin{gathered}
\left|\Delta(z, \eta)-\Delta_{m}(z, \eta)\right|= \\
=\left|\int_{a}^{b} f\left(s, x_{\infty}(s, z, \eta), \frac{d x_{\infty}(s, z, \eta)}{d t}\right) d s-\int_{a}^{b} f\left(s, x_{m}(s, z, \eta), \frac{d x_{m}(s, z, \eta)}{d t}\right) d s\right| \leq \\
\leq K \int_{a}^{b} \frac{10}{9} \alpha_{1}(s, a, b) Q^{m}\left(1_{n}-Q\right)^{-1} \delta_{[a, b], D, D_{1}}(f) d s= \\
=\frac{10(b-a)^{2}}{27} K Q^{m}\left(1_{n}-Q\right)^{-1} \delta_{[a, b], D, D_{1}}(f),
\end{gathered}
$$

which proves (2.11).

From (2.9) and (2.10) using the Lipschitz conditions (1.4), (1.5) and estimates (2.7), (2.8), we obtain

$$
\begin{gathered}
\left.\left|\Lambda(z, \eta)-\Lambda_{m}(z, \eta)\right|=\mid g\left(x_{\infty}(a, z, \eta), x_{\infty}(b, z, \eta), \int_{a}^{b} h\left(s, x_{\infty}(b, z, \eta)\right) d s\right)\right)- \\
\quad-g\left(x_{m}(a, z, \eta), x_{m}(b, z, \eta), \int_{a}^{b} h\left(s, x_{m}(s, z, \eta)\right) d s\right) \mid \leq \\
\leq K_{3}\left|x_{\infty}(a, z, \eta)-x_{m}(a, z, \eta)\right|+K_{4}\left|x_{\infty}(b, z, \eta)-x_{m}(b, z, \eta)\right|+
\end{gathered}
$$




$$
\begin{gathered}
+(b-a) K_{5} K_{6}\left|x_{\infty}(t, z, \eta)-x_{m}(t, z, \eta)\right| \leq \\
\leq \frac{5(b-a)}{9}\left[K_{3}+K_{4}+(b-a) K_{5} K_{6}\right] Q^{m}\left(1_{n}-Q\right)^{-1} \delta_{[a, b], D, D_{1}}(f),
\end{gathered}
$$

i.e. (2.12) holds also.

Based on both exact and approximate determining systems (2.9) and (2.10) let us introduce the mappings $H: D_{a} \times D_{b} \rightarrow \mathbb{R}^{2 n}$ and $H_{m}: D_{a} \times D_{b} \rightarrow \mathbb{R}^{2 n}$ by setting

$$
\begin{gathered}
H(z, \eta)=\left[\begin{array}{c}
{[\eta-z]-\int_{a}^{b} f\left(s, x_{\infty}(a, z, \eta), x_{\infty}(b, z, \eta), \int_{a}^{b} h\left(s, x_{\infty}(s, z, \eta)\right)\right) d s} \\
g\left(x_{\infty}(a, z, \eta), x_{\infty}(b, z, \eta), \int_{a}^{b} h\left(s, x_{\infty}(b, z, \eta)\right) d s\right)-d
\end{array}\right. \\
H_{m}(z, \eta)=\left[\begin{array}{c}
{[\eta-z]-\int_{a}^{b} f\left(s, x_{m}(a, z, \eta), x_{m}(b, z, \eta), \int_{a}^{b} h\left(s, x_{m}(s, z, \eta)\right)\right) d s} \\
g\left(x_{m}(a, z, \eta), x_{m}(b, z, \eta), \int_{a}^{b} h\left(s, x_{m}(b, z, \eta)\right) d s\right)-d
\end{array}\right.
\end{gathered}
$$

$(z, \eta) \in D_{a} \times D_{b}$. We see from Theorem 2 that the critical points of the vector field $H$ of the form (2.13) determine solutions of the non-linear boundary value problem (1.1)-(1.2). The next statement establishes a similar result based upon properties of vector field $H_{m}$ explicity known from (2.14).

Theorem 3. Assume that the conditions of Lemma 1 hold. Moreover, one can specify an $m \geq 1$ and a set

$$
\Gamma:=D_{1} \times D_{2} \subset \mathbb{R}^{2 n}
$$

where $D_{1} \subset D_{a}, D_{2} \subset D_{b}$ are certain bounded open sets such that the mapping $H_{m}$ satisfies the relation

$$
\left|H_{m}(z, \eta)\right| \triangleright_{\partial \Gamma}\left[\begin{array}{c}
\frac{10(b-a)^{2}}{27} K Q^{m}\left(1_{n}-Q\right)^{-1} \delta_{[a, b], D, D_{1}}(f) \\
\frac{5(b-a)}{9}\left[K_{3}+K_{4}+(b-a) K_{5} K_{6}\right] Q^{m}\left(1_{n}-Q\right)^{-1} \delta_{[a, b], D, D_{1}}(f)
\end{array}\right]
$$

on the boundary $\partial \Gamma$ of the set $\Omega$. If, in addition

$$
\operatorname{deg}\left(H_{m}, \Omega, 0\right) \neq 0,
$$

then there exists a pair $\left(z^{*}, \eta^{*}\right) \in D_{1} \times D_{2}$ for which the function

$$
x^{*}(\cdot):=x_{\infty}\left(\cdot, z^{*}, \eta^{*}\right)
$$

is a solution of the non-linear boundary value problem (1.1)-(1.2). 
In (2.15) the binary relation $\triangleright_{\partial \Gamma}$ is defined in [1] as a kind of strict inequality for vector functions and it means that at every point on the boundary $\partial \Gamma$ at least one of the components of the vector $\left|H_{m}(z, \eta)\right|$ is greater than the corresponding component of the vector on the right-hand side. The degree in (2.16) is the Brouwer degree because all the vectors fields are finite-dimensional. Likewise, all the terms on the right-hand side of (2.15) are computed explicitly e.g. by using computer algebra system.

Proof. The proof can be carried out similarly as in Theorem 4 from [3].

\section{EXAMPLE}

Let us apply the approach described above to the system of differential equations

$$
\left\{\begin{array}{l}
\frac{d x_{1}(t)}{d t}=\frac{1}{2} x_{2}^{2}(t)-t \frac{d x_{2}(t)}{d t} x_{1}(t)+\frac{1}{32} t^{3}-\frac{1}{32} t^{2}+\frac{9}{40} t \\
\frac{d x_{2}(t)}{d t}=\frac{1}{2} \frac{d x_{1}(t)}{d t} x_{1}(t)-t^{2} x_{2}(t)+\frac{15}{64} t^{3}+\frac{1}{80} t+\frac{1}{4}
\end{array}, t \in[0,1],\right.
$$

considered with non-linear boundary conditions

$$
\begin{aligned}
x_{1}(0) x_{2}(1)+\left[\int_{0}^{1} x_{1}(s) d s\right]^{2} & =-\frac{311}{14400}, \\
x_{1}(1) x_{2}(0)-\int_{0}^{1} x_{2}(s) d s & =-\frac{1}{8} .
\end{aligned}
$$

Introduce the vector of parameters $z=\operatorname{col}\left(z_{1}, z_{2}\right), \eta=\operatorname{col}\left(\eta_{1}, \eta_{2}\right)$. Let us consider the following choice of the subsets $D_{a}, D_{b}$ and $D^{1}$ :

$$
\begin{gathered}
D_{a}=D_{b}=\left\{\left(x_{1}, x_{2}\right):-0.1 \leq x_{1} \leq 0.2,-0.2 \leq x_{2} \leq 0.3\right\}, \\
D^{1}=\left\{\left(\frac{d x_{1}}{d t}, \frac{d x_{2}}{d t}\right):-0.1 \leq \frac{d x_{1}}{d t} \leq 0.3,-0.1 \leq \frac{d x_{2}}{d t} \leq 0.3\right\} .
\end{gathered}
$$

In this case $D_{a, b}=D_{a}=D_{b}$. For $\rho=\operatorname{col}\left(\rho_{1}, \rho_{2}\right)$ involved in (1.12), we choose the vector $\rho=\operatorname{col}(0.4 ; 0.4)$. Then, in view of $(2.13)$ the sets $(1.8)$ and $D_{2}$ takes the form:

$$
D=D_{\rho}=\left\{\left(x_{1}, x_{2}\right):-0.5 \leq x_{1} \leq 0.6,-0.6 \leq x_{2} \leq 0.7\right\}
$$

and

$$
D_{2}=\left\{\left(x_{1}, x_{2}\right): 0.25 \leq x_{1} \leq 0.36,-0.6 \leq x_{2} \leq 0.7\right\} .
$$

A direct computation shows that the conditions (1.3)-(1.5) hold with

$$
\begin{gathered}
K_{1}=\left[\begin{array}{cc}
0.3 & 0.7 \\
0.15 & 1
\end{array}\right], K_{2}=\left[\begin{array}{cc}
0 & 0.6 \\
0.3 & 0
\end{array}\right], K_{3}=\left[\begin{array}{cc}
0.3 & 0 \\
0 & 0.2
\end{array}\right], \\
K_{4}=\left[\begin{array}{cc}
0 & 0.2 \\
0.3 & 0
\end{array}\right], K_{5}=\left[\begin{array}{cc}
1.2 & 0 \\
0 & 1
\end{array}\right], K_{6}=\left[\begin{array}{ll}
1 & 0 \\
0 & 1
\end{array}\right]
\end{gathered}
$$


TABLE 1.

\begin{tabular}{|c|r|r|r|r|}
\hline $\mathrm{m}$ & $z_{1}$ & $z_{2}$ & $\eta_{1}$ & $\eta_{2}$ \\
\hline 0 & -0.089643967 & -0.0002812586 & 0.03176891 & 0.25026338 \\
\hline 1 & -0.0994489263 & 0.00051937347 & 0.0255001973 & 0.2504687527 \\
\hline 4 & -0.0999998827 & $7.744981 \cdot 10^{-8}$ & 0.02500007591 & 0.25000011 \\
\hline 6 & -0.1000000004 & $-2.263731 \cdot 10^{-10}$ & 0.02499999973 & 0.2499999996 \\
\hline Exact & -0.1 & 0 & 0.025 & 0.25 \\
\hline
\end{tabular}

and therefore $r\left(K_{2}\right)=\sqrt{0.18}<1$, and in (1.10) the matrix

$$
\begin{gathered}
K=\left[\begin{array}{cc}
0.4756097561 & 1.585365854 \\
0 . .2926829268 & 1.475609756
\end{array}\right], \\
Q=\left[\begin{array}{ll}
0.07134146342 & 0.2378048781 \\
0.04390243902 & 0.2213414634
\end{array}\right], r(Q)=0.273090089272152<1 .
\end{gathered}
$$

Furthermore, in view of (1.11)

$$
\begin{gathered}
\delta_{[a, b], D, D^{1}}(f):=\frac{1}{2}\left[\max _{(t, x, y) \in[a, b] \times D \times D^{1}} f(t, x, y)-\min _{(t, x, y) \in[a, b] \times D \times D^{1}} f(t, x, y)\right]= \\
=\left[\begin{array}{c}
0.31 \\
0.7325
\end{array}\right], \\
\rho=\left[\begin{array}{c}
0.4 \\
0.4
\end{array}\right] \geq \frac{b-a}{2} \delta_{[a, b], D, D^{1}}(f)=\left[\begin{array}{c}
0.155 \\
0.36625
\end{array}\right] .
\end{gathered}
$$

We thus see that all conditions of Theorem 1 are fulfilled, and the sequence of functions (2.2) for this example is uniformly convergent.

Applying Maple 14, we can carried out the calculations.

It is easy to check that

$$
x_{1}^{*}(t)=\frac{t^{2}}{8}-\frac{1}{10}, \quad x_{2}^{*}(t)=\frac{t}{4}
$$

is an exact continuously differentiable solution of the problem (1.1), (1.2). For a different number of approximations $m$ we obtain from (2.10) the following numerical values for the introduced parameters, which are presented in Table 3.

On the Figure 1 one can see the graphs of the exact solution (solid line) and its zero $(\diamond)$ and sixth approximation $(\times)$ for the first and second coordinates.

The error of the sixth approximation $(m=6)$ for the first and second components:

$$
\max _{t \in[0,1]}\left|x_{1}^{*}(t)-x_{61}(t)\right| \leq 1 \cdot 10^{-9}, \max _{t \in[0,1]}\left|x_{2}^{*}(t)-x_{62}(t)\right| \leq 5 \cdot 10^{-9} .
$$



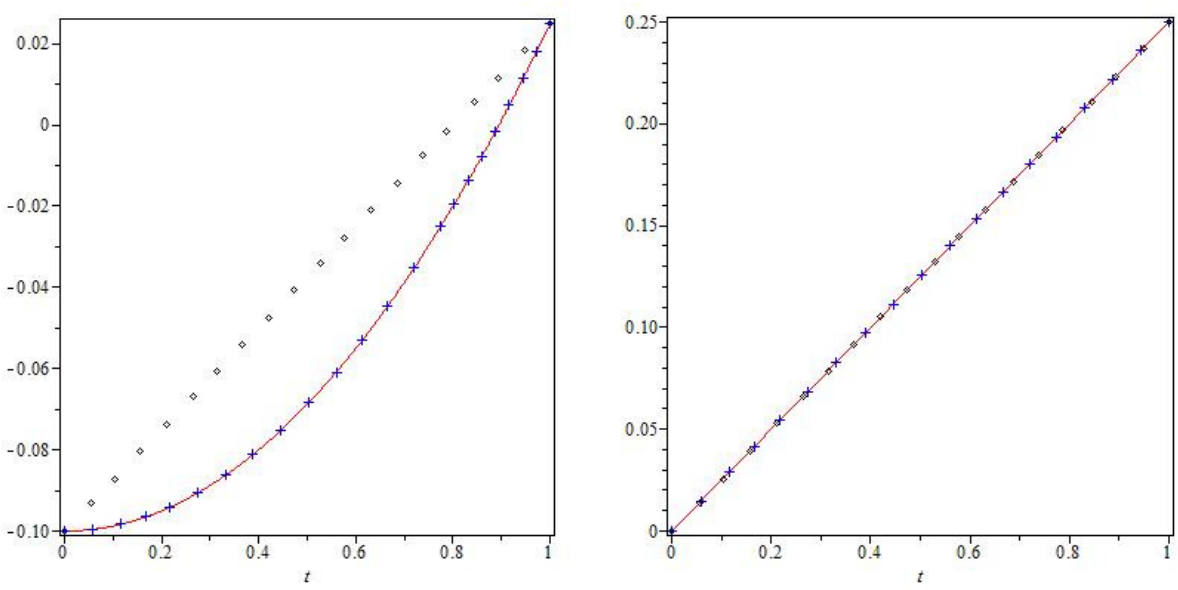

FIGURE 1.

\section{REFERENCES}

[1] A. Rontó and M. Rontó, "Successive approximation techniques in non-linear boundary value problems for ordinary differential equations," in Handbook of differential equations: ordinary differential equations. Vol. IV, ser. Handb. Differ. Equ. Elsevier/North-Holland, Amsterdam, 2008, pp. 441-592.

[2] A. Rontó, M. Rontó, and J. Varha, "A new approach to non-local boundary value problems for ordinary differential systems," Applied Mathematics and Computation, vol. 250, pp. 689-700, 2015, doi: http://dx.doi.org/10.1016/j.amc.2014.11.021.

[3] M. Rontó and Y. Varha, "Constructive existence analysis of solutions of non-linear integral boundary value problems," Miskolc Math. Notes, vol. 15, no. 2, pp. 725-742, 2014, doi: 10.18514/MMN.2014.1319.

[4] M. Rontó and Y. Varha, "Successive approximations and interval halving for integral boundary value problems," Miskolc Math. Notes, vol. 16, no. 2, pp. 1129-1152, 2015, doi: 10.18514/MMN.2015.1708.

[5] M. Rontó, Y. Varha, and K. Marynets, "Further results on the investigation of solutions of integral boundary value problems," Tatra Mt. Math. Publ., vol. 63, pp. 247-267, 2015, doi: 10515/tmmp2015-0035.

Author's address

\section{Varga}

Mathematical Faculty of Uzhhorod National University, 14 Universitetska St., 88000, Uzhhorod, Ukraine

E-mail address: iana.varga@uzhnu.edu.ua 